\title{
COSTOS DE NÓMINA NO IDENTIFICADOS POR FASE EN CONSTRUCCIÓN DE OBRA EJECUTADA NO SUBCONTRATADA CON TERCEROS
}

\section{PAYROLL COSTS NOT IDENTIFIED BY PHASE IN CONSTRUCTION WORK DONE WITH NO THIRD SUBCONTRACTED}

\begin{abstract}
Rubén Antonio González Franco1; Jesús Manuel Meza-Valdez2 y Rubén Miranda López3
Profesor e investigador de tiempo completo titular "B" y Coordinador General de Investigación y Posgrado de la Facultad de Contaduría y Administración de la Universidad Autónoma de Sinaloa, Campus Culiacán1. Profesor de cátedra de Instituto Tecnológico de Estudios Superiores Campus Culiacán, de Tec Milenio Campus Culiacán, de Universidad de Durango y de la Facultad de Contaduría y Administración2. Profesor e investigador de tiempo completo titular "C" Director de la Facultad de Contaduría y Administración de la Universidad Autónoma de Sinaloa, Campus Culiacán, miembro del cuerpo académico en Formación "Estudios Fiscales y Administrativos" UAS-CA-2563.
\end{abstract}

\section{RESUMEN}

El Reglamento del Seguro Social aplicable a las empresas de la Construcción en lo referente al cumplimiento de alta de Obra obliga al propietario a generar una alta por cada etapa de construcción y por consiguiente a comprobar los montos de materiales, maquinaria y equipo, así como de mano de obra aplicados en la misma etapa, todo con la finalidad de validar que se cumple $100 \%$ con el pago de las cuotas obrero-patronales por parte del patrón, lineamientos que en el caso especifico de las PYMES se vuelve una práctica más compleja, pues los controles que dichas empresas tienen no permiten comprobar los costos por etapas en dos rubros en especial, como son los costos ejercidos de materiales y mano de obra por vivienda, motivo por el cual esta investigación pretende resolver la problemática de generar lineamientos claros para poder cumplir con la comprobación de pago de las cuotas en el rubro de mano de obra cuando se ejerce de manera directa, y a través de la aplicación de entrevistas directas y cuestionarios a las unidades de análisis conformada por empresarios, representantes, directores o contadores de empresas PYMES de la construcción, personal de la Cámara Nacional de la Industria de la Construcción, y del Instituto Mexicano del Seguro Social responsable del departamento de Construcción, quienes darán respuesta a las preguntas que el mismo instrumento contempla, y con esto se logró determinar que en efecto la percepción de estas PYMES es que se requiere dejar un criterio estándar para su comprobación y el más apropiado sería el del prorrateo de costos de manera proporcional en base a las fechas de contratación y fechas de término de las obras llevando un control que identifique costos de manera global, dejando un número de identificación en los papeles de trabajo para futuras revisiones por parte de la autoridad.

Palabras clave: Constructora, SATIC, Alta de Obra, AFIL Obra IMSS, Vivienda

\begin{abstract}
The Social Insurance Regulations applicable to the construction companies with regard to meeting high work requires the owner to generate high for each construction phase and thus to check the amounts of materials, machinery and equipment, as
\end{abstract}


well as labor applied at the same stage, all in order to validate that it complies $100 \%$ with the payment of worker-employer contributions by the employer, guidelines in the specific case of SMEs practice becomes more complex, controls as these companies have no proof phased costs in two areas in particular, as are exercised costs of materials and workmanship for housing, which is why this research aims to solve the problem of generating clear guidelines to comply with the verification of payment of contributions in the field of labor when it is exercised directly, and through the application of direct interviews and questionnaires to the units of analysis consists of businessmen, representatives, directors or business accountants SMEs construction, staff of the National Chamber of construction Industry and the Mexican Social Security Institute head of the Department of construction, who will answer questions the same instrument provides, and with this it was determined that in fact the perception of these SMEs is required to leave a standard criterion for testing and would be more appropriate apportionment of costs proportionately based on hire dates and termination dates of the work keeping track of costs identifying overall, leaving an identification number in the working papers for further review by the authority.

Keywords: Construction, SATIC, High Force, AFIL IMSS Works, Housing.

\section{INTRODUCCIÓN}

Antecedentes, los proyectos de construcción son indispensables para el desarrollo moderno, equilibrado y sustentable de cualquier país; estos proyectos generan infraestructura física que cada vez es más compleja y costosa. Es importante considerar la estructura y la organización de la industria de la construcción en México y lo confirmamos con criterios de la Cámara Mexicana de la Industria de la Construcción (González, 2010.+ p.6 y 7), al señalar que el 93\% de las firmas están clasificadas como tal.

Las PyMES generan a nivel mundial más del $90 \%$ del negocio y son responsables del $50 \%$ al $60 \%$ de los empleos. En Latinoamérica el $95 \%$ de las firmas son PyMES y generan entre el $40 \%$ y $60 \%$ de la oferta laboral contribuyendo entre el $30 \%$ y $50 \%$ del producto interno bruto. Por otro lado en Estados Unidos el 99.7\% de todas las firmas cayeron en la categoría de "pequeños negocios" y contribuyen en más del $50 \%$ del Producto Interno Bruto, así mismo de acuerdo a informe de la OECD (2005) indica que en la mayoría de economías las PyMES generan dos tercios del empleo del sector privado y son la fuente principal para la creación de empleos.

Con el siguiente cuadro vemos de manera concentrada los criterios que se tienen en varios países para definir el tipo de empresas PYMES de acuerdo al número de empleados, así pues en Francia existen cinco categorías, EUA tiene sólo tres al igual que la CEPAL, pero en México se has establecido cuatro, de acuerdo a datos proporcionados por la Secretaría de Economía. El tipo de empresa considerada PyME en México, puede llegar a ser artesanal y micro para Francia, mediana en EUA, y pequeña para la CEPAL, por lo que es menester cuidar cuando se hable de PYMES en cada uno de estos países por representar diferencias tan significativas.

Este tipo de empresas viven una problemática constante pues día a día enfrentan una lucha por la supervivencia, crecimiento, desarrollo, competitividad, búsqueda de estrategias que le ayuden a minimizar la carga que actualmente tienen y que repercute 


\section{Cuadro 1. Criterios con los que se definen a las PYMES de acuerdo al número de Empleados}

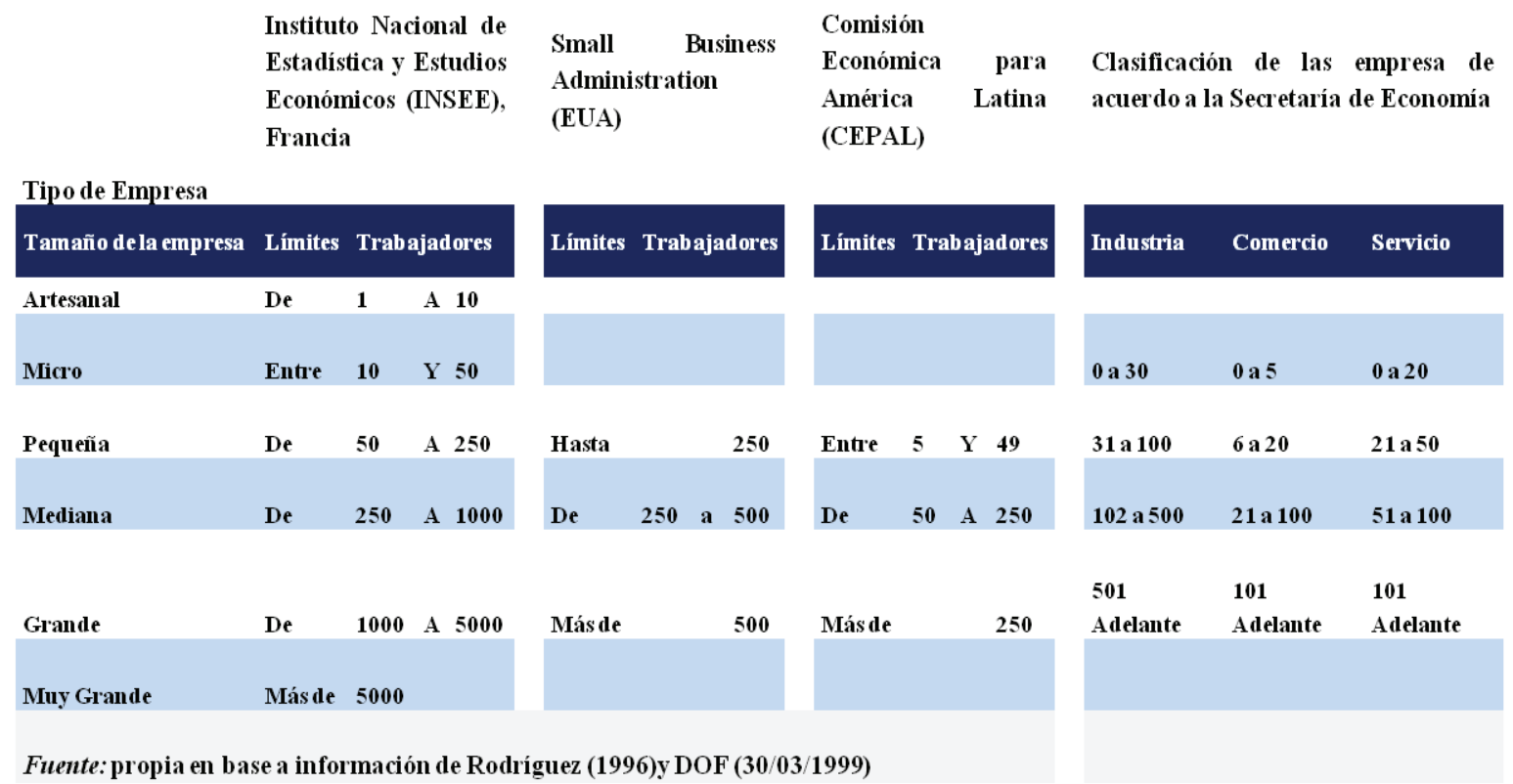

directamente en las variables anteriormente señaladas y sobre todo en su rentabilidad.

El problema Objeto de Estudio

El Reglamento del Seguro Social obligatorio para los trabajadores de la construcción por obra o tiempo determinado, señala la obligación para las empresas que se dedican a este giro de generar las altas y movimientos de obras adicionales mediante los formatos conocidos como SATIC, anteriormente identificados como AFIL. Dichos formatos se sustentan con los contratos firmados entre los propietarios de las obras (que en algunas veces no corresponde a la misma que tiene el giro de construcción, primer problema que se presenta para identificar quién dará las altas), y el subcontratista; adicionando el formato de alta o movimientos de obra exigido a dichos subcontratistas, pues con esto se obligan a cumplir con los pagos de cuotas de seguridad social, aún y cuando se señala la obligación solidaria de pago de las cuotas obrero patronales.

Los tres rubros que contemplan los contratos son: materiales, maquinaria y equipo, y mano de obra, datos que se detallarán en los formatos de SATIC-01, con sus respectivos anexos. Contar con el contrato firmado y presentando el aviso al Instituto de la subcontratación de parte o partes de la obra dentro de los cinco días hábiles siguientes libera de responsabilidad del pago de las cuotas obrero-patronales a las empresas contratantes, por lo mismo es menester contar con el contrato firmado en original, para presentación al IMSS en caso de ser necesario.

Poder comprobar a las autoridades el cumplimiento de pago de cuotas, es un proceso complicado, pues la parte ejercida de obra directa debemos identificarla también en los rubros de materiales y mano de obra por cada formato de SATIC dado de alta y, considerando que el mercado de vivienda y las condiciones macroeconómicas generan cambios en los proyectos de construcción, a lo cual se suma que los sistemas de información con los que cuentan las PYMES no permite identificar la mano de obra por 
cada vivienda, pues la nómina se elabora por todo el personal independiente de la fase en la cual se encuentra desarrollando sus actividades, resulta pertinente esta investigación para generar las condiciones necesarias entre las empresas de construcción del sector vivienda y el IMSS; y con esto evitar la aplicación de sanciones a las empresas por no poder presentar los costos por cada una de las viviendas que los contratos contemplan en las etapas de alta generadas, buscando con esto el amarre entre inversión efectuada y pago de cuotas.

Desde el año 2010 el Instituto Mexicano del Seguro Social en el afán de recaudación de flujo a través del pago de cuotas y ejerciendo sus facultades de comprobación, exige a los contribuyentes que generaron la SATIC01 a cubrir las cuotas no pagadas por los subcontratistas, situación que está en contra de lo señalado en el Reglamento mencionado, y extralimitando sus facultades exige el pago con la presión moral de cierre de la obra en construcción, situación que implica pérdidas muy significativas para los contribuyentes.

Las negociaciones en este sentido estriban en al menos entregar al Instituto las cantidades retenidas a los contratistas a cuenta del Fondo de Garantía y no devuelto por falta del finiquito de obra, cuya diligencia se está volviendo una práctica constante, pues de esta manera el IMSS garantiza al menos una parte de los adeudos del contribuyente final.

\section{MATERIALES Y MÉTODOS}

La presente investigación se ubica en el ámbito de las ciencias sociales por que su objetivo principal ha sido diseñado para medir el impacto que generaron los cambios en el reglamento del IMSS en lo referente a la problemática para comprobar al Instituto Mexicano del Seguro Social el cumplimiento detallado del pago de las contribuciones de seguridad social para los trabajadores eventuales que en dichas obras se desempeñan.

Por considerar de sumo interés conocer las características más importantes de las PyMES mexicanas, a continuación se presenta un estudio realizado por la doctora Pilar Baptista, citado en Rodríguez (2001, p. 50), en donde se muestra la compleja situación de las empresas mexicanas.

Deacuerdoconlosresultados deinvestigación de la doctora Pilar Baptista, se puede asumir que la problemática y oportunidades de la pequeña y mediana empresa se generan de las características del propietario, ya que se da una relación de mutua dependencia en donde sus cualidades y defectos se reflejan en la empresa que dirige. Además, la falta de conocimientos técnicos sobre administración da lugar a consecuencias considerablemente más graves en este tipo de empresa que en las grandes compañías, visto desde la perspectiva económica. Ello supone que la empresa pequeña y mediana depende, esencialmente, de la calidad de su dirigente, lo que pone en riesgo la permanencia de la misma en ausencia de él. Además de esto, la falta de claridad que las mismas leyes y reglamentos contemplan, generan en los administradores el no contar con los elementos para poder hacer frente a las revisiones de las autoridades, situación que se percibe y se aprecia en la ausencia de control interno que sumado a la falta de sistemas informáticos clave puedan lograr el control de los costos de mano de obra en las altas de etapas de obra de construcción. (Rodríguez, et al. 2001)

Área de Estudio

América Latina y en especial México 
tienen en la empresa familiar el principal motor de sus economías, representando más del 80\% de las empresas de la región. La empresa familiar dota de la mayor cantidad de empleo a la población y desde su seno se generan y reproducen los marcos institucionales del comportamiento de gran parte de la población. Este tipo de empresa impacta de manera importante no sólo en el ámbito económico ya referenciado, sino en lo social, cultural, político y psicológico de dichas sociedades (Lozano, Cisneros y Niebla, 2009, p. 17).
Como lo establece el Plan Nacional de Desarrollo 2007-2012, el Desarrollo Humano Sustentable es el objetivo rector de la presente administración. Es por ello que las políticas públicas del Gobierno Federal se orientan a establecer condiciones que permitan a toda la población incrementar sus capacidades, así como ampliar las oportunidades tanto para las generaciones presentes como las futuras, es decir, se busca que todos los mexicanos tengan una vida digna sin comprometer el bienestar de las siguientes generaciones.

\section{Cuadro 2. Perfil de la empresa pequeña y mediana en México}

\begin{tabular}{|c|c|}
\hline Factores & Resultados \\
\hline Datos estructurales & $\begin{array}{l}\text { Su estructura orgánica es familiar, y existen problemas para su clasificación en } \\
\text { tamaños }\end{array}$ \\
\hline Tipos de dirigentes & $\begin{array}{l}\text { El típico director es un hombre de } 44 \text { años, con catorce años de escolaridad y es } \\
\text { empresario por herencia. Tiene problemas de tipo familiar en la administración } \\
\text { y muchas de éstas desaparecen con su fundador. }\end{array}$ \\
\hline Tecnología & La mayor parte utiliza sistemas de producción tradicional. \\
\hline Medio Ambiente & $\begin{array}{l}\text { Su medio ambiente es complejo y sostienen relaciones con otras organizaciones: } \\
\text { proveedores, clientes, competencias, asociaciones, bancos, gobierno y despachos } \\
\text { profesionales de contaduría. }\end{array}$ \\
\hline Valores y objetivos & $\begin{array}{l}\text { Para la mayoría de los directivos el objetivo principal es maximizar las } \\
\text { utilidades. Los procedimientos administrativos son sumamente personalizados, } \\
\text { es decir, existe la vigilancia estrecha sobre las operaciones. La toma de } \\
\text { decisiones es centralizada y la ejecución la realizan jefes de área, a quienes se les } \\
\text { responsabiliza por los resultados obtenidos. }\end{array}$ \\
\hline $\begin{array}{l}\text { Problemática } \\
\text { enfrentan }\end{array}$ & $\begin{array}{l}\text { En órden de importancia, son ocho problemas que a juicio de los empresarios } \\
\text { son los más relevantes en las decisiones: recursos humanos, deficiencias del } \\
\text { gobierno, falta de seguridad con los proveedores, financiamiento, materias } \\
\text { primas (calidad y escasez), mercados, competencias y deficiente organización. }\end{array}$ \\
\hline Planeación y crecimiento & $\begin{array}{l}\text { La mayoría de las empresas han crecido en los últimos años. Un cuarenta por } \\
\text { ciento de los entrevistados señaló no querer crecer más; la razón, que se les } \\
\text { escape el control de su empresa. En lo que respecta a planeación estratégica es } \\
\text { casi inexistente. }\end{array}$ \\
\hline Procesos informativos & $\begin{array}{l}\text { Lo relevante es estar al día en cuanto a los productos y tecnología. Los datos los } \\
\text { obtienen mediante visitas al extranjero, revistas especializadas, información de } \\
\text { sus clientes. Sobre técnicas administrativas, las adquieren de cursos externos. } \\
\text { Los aspectos financieros los obtienen con otrosindustriales y banqueros. }\end{array}$ \\
\hline
\end{tabular}

Fuente. Elaboración propia en base a Pilar Baptista, citado en Rodríguez (2001, p. 50).

La empresa familiar es una forma de organización compleja. En dicha organización la empresa, la familia y el patrimonio se empalman, se confrontan, se enriquecen o se destruyen. 
Pero el logro de una vida digna debe abarcar aspectos que el mismo Gobierno tome en cuenta al definir y establecer leyes y reglamentos en el rubro de seguridad social como en otros impuestos; dicha normatividad debe aplicarse por las entidades fiscalizadoras con efectividad y claridad (no dando lugar a la subjetividad de cumplimiento). Lo antes señalado se vincula con lo mencionado por Rojas (2002, p. 15), es uno de los factores que contribuye a la necesidad de reformas constantes en los países, pues lainsuficiencia de contribuciones de impuestos de los contribuyentes en los diferentes rubros o la corrección a las misma reglamentación para dejar claro la forma completa de operar dichas reglamentaciones evitando que los contribuyentes incumplan con el pago o simplemente no cuenten con las líneas claras para ejercer el derecho de comprobación de los pagos efectuados y explica Rojas (2003, p.18) que entre mayor sea el monto que el gobierno en España destina a educación de alto nivel mayor es el monto que las empresas participan en sus costos de seguridad social, pues esto nos debe impulsar a buscar que la recaudación en México sea más eficiente día con día para lograr su efecto multiplicador en los otros indicadores nacionales.

En particular, la política económica se ha dirigido a impulsar la competitividad del país, a fin de promover un mayor crecimiento económico e incrementar la generación de empleos, de eliminar los obstáculos que impiden reducir de manera permanente la pobreza, y así alcanzar un mayor nivel de desarrollo humano sustentable. Por un lado, se alienta el fortalecimiento de los factores transversales como el Estado de Derecho y la seguridad pública; la infraestructura y la formación de capital humano; el desarrollo tecnológico; la promoción de la competencia; y un marco regulatorio que atraiga la inversión. Por otra parte, se estableció una agenda sectorial en la que se reconoce la necesidad de alentar industrias clave para detonar el crecimiento económico, como: sector agropecuario, construcción (especialmente de vivienda e infraestructura de comunicaciones $\mathrm{y}$ transportes), turismo, financiero $\mathrm{y}$ manufacturero.

De acuerdo al Documento de trabajo número 65 del Centro de Estudios Sociales y de Opinión Pública de marzo del 2009, en virtud de que la construcción de vivienda es considerada una actividad de primera importancia para el desarrollo nacional por diversas razones y dentro del plano económico se reconoce su impacto sobre la inversión, la producción y el empleo, así como sobre los niveles de bienestar de las familias; y por otro lado en el plano político porque, por una parte, ha motivado el diseño y aplicación de políticas específicas que han fortalecido y legitimado la acción gubernamental, sobre todo porque desde 1983 el derecho a la vivienda ha sido elevado a rango constitucional, por estos motivos también el Gobierno debe buscar generar las condiciones necesarias para impulsar a las PYMES de la construcción hacia el cumplimiento del ciclo completo de atención a lo que señala el Reglamento en mención.

En congruencia con lo anterior, el área objeto de estudio de esta investigación comprende el municipio de Culiacán, por corresponder al lugar en el cual se encuentran una de las empresas desarrolladoras mexicanas de vivienda que han logrado un crecimiento sostenido muy fuerte, logrando después del Distrito Federal (con 43 empresas constructoras) el segundo lugar de las 100 empresas Constructoras más importantes en México (Ver Cuadro No. 3), considerando su nivel de ingresos, generando por sí sola 
un $9 \%$ de los ingresos totales que las 100 Constructoras generaron, y el $8 \%$ de los empleos generados por esta industria en nuestro país y esto debe ser un ejemplo claro para las demás empresas del mismo giro que tenemos en este municipio para lograr eficiencia por medio de programas de mejora de la calidad y de convertirse en empresas con crecimiento.

Con esta filosofía pretendemos generar condiciones para que las PYMES puedan cumplir con sus principales obligaciones preliminares como el alta de sus obras, el pago de las contribuciones en materia de seguridad social y cerrando el ciclo con la comprobación de dichos pagos vinculadas con cada una de las altas de etapa de sus obras; y esto sabemos, permitirá cumplir con el objetivo que el Gobierno tiene establecido en mejora de los indicadores económicos de la localidad, de la región y del país. En forma adicional y en sustento a los mismos indicadores tenemos el impacto social que genera en la calidad de vida de los trabajadores que en ella laboran, y en el resto de la sociedad por tener acceso a vivienda para sus familias.

El municipio de Culiacán se encuentra en la región central del estado de Sinaloa, entre los meridianos $106^{\circ} 56^{\prime} 50^{\prime}$ y $107^{\circ}$ 50 ' 15 " de longitud oeste del meridiano de Greenwich y las coordenadas extremas de los paralelos $24^{\circ} 02^{\prime} 10^{\prime \prime}$ y $25^{\circ} 14^{\prime} 56^{\prime \prime}$ de latitud norte. Su altitud sobre el nivel del mar en la costa alcanza hasta los 2,100 metros en la zona de los altos. Su cabecera municipal tiene una altura media de 53 metros sobre el nivel del mar. Colinda al norte con el municipio de Badiraguato, al sur con el golfo de California, al este con el municipio de Cosalá y el estado de Durango; al oeste con el municipio de Navolato; al noroeste con el estado de Durango; al noroeste con
Navolato y Mocorito; al suroeste con Elota y Cosalá, y al suroeste con Navolato y el Golfo de California. La problemática habitacional en el área urbana presenta como factores la especulación de lotes y fincas, la irregularidad en la tenencia y proliferación de nuevos asentamientos en la zona periférica donde la construcción es precaria. En Culiacán la mayoría de las viviendas cuentan con los servicios básicos de electricidad, agua potable, alcantarillado y drenaje. El tipo de construcción es de concreto, sin mostrar ningún rasgo arquitectónico especial. Las edificaciones más antiguas están construidas de adobe crudo, cuyos techos se encuentran sostenidos con vigas de madera, principalmente localizadas en el Centro Histórico de esta ciudad capital.

Las principales actividades desarrolladas por las 10 primeras empresas, de las 21 señaladas en el Catálogo del cuadro No. 4., se componen de las actividad 1 (Holding), actividad 2 (Vivienda económica y de interés social), actividad 3 (Vivienda media y residencial), actividad 4 (Edificación comercial, oficinas y servicios), y actividad 5 (Edificación de naves y plantas industriales), por tal motivo se sustenta esta investigación en las empresas PYMES, las cuales enfocan sus esfuerzos y recursos en la mayor parte de construcción de vivienda económica y de interés social.

Al analizar las actividades que las 100 Constructoras más importantes desarrollaron nos damos cuenta que en suma son 392 actividades las desarrolladas considerando que tenemos empresas que su giro completo va desde una actividad, hasta llegar al máximo donde una sola empresa lleva a cabo dieciséis actividades de las veintiún que el catálogo anterior contempla. 
Cuadro 3. Empresas por Estado y su relación Ingresos-Posición.

\begin{tabular}{|c|c|c|c|c|}
\hline & & & $\%$ & \\
\hline Lugar & Estados & Ingresos MDP & Ing. & No. Empresas \\
\hline \multirow[t]{2}{*}{3} & Baja California & 16359.1 & $8 \%$ & 6 \\
\hline & \multicolumn{2}{|c|}{ Baja California } & & \\
\hline 5 & SUR & 2293.7 & $1 \%$ & 2 \\
\hline 5 & Campeche & 1684.3 & $1 \%$ & 2 \\
\hline 4 & Chihuahua & 7285.1 & $3 \%$ & 4 \\
\hline 6 & Coahuila & 315.7 & $0 \%$ & 1 \\
\hline 1 & Distrito Federal & 143051.5 & $67 \%$ & 43 \\
\hline 6 & Durango & 185.9 & $0 \%$ & 1 \\
\hline 6 & Guanajuato & 111.1 & $0 \%$ & 2 \\
\hline 6 & Guerrero & 439.8 & $0 \%$ & 1 \\
\hline 6 & Hidalgo & 438 & $0 \%$ & 1 \\
\hline 5 & Jalisco & 2116.4 & $1 \%$ & 8 \\
\hline 5 & México & 1936.2 & $1 \%$ & 6 \\
\hline 3 & Nuevo León & 16052 & $8 \%$ & 10 \\
\hline 6 & Puebla & 369.2 & $0 \%$ & 3 \\
\hline \multirow{2}{*}{$\begin{array}{l}6 \\
6\end{array}$} & Querétaro & 882.2 & $0 \%$ & 2 \\
\hline & Quintana Roo & 89.9 & $0 \%$ & 1 \\
\hline \multirow[t]{2}{*}{6} & San Luis Potosí & 220.9 & $0 \%$ & 2 \\
\hline & \multicolumn{2}{|l|}{ Sinaloa } & & \\
\hline 2 & $C U L I A C A N$ & 18850.5 & $9 \%$ & 1 \\
\hline 6 & Veracruz & 537.7 & $0 \%$ & 3 \\
\hline 6 & Yucatán & 719.4 & $0 \%$ & 1 \\
\hline & Totalgeneral & 213938.6 & $100 \%$ & 100 \\
\hline
\end{tabular}

Fuente. Propia elaborada con base en los datos generados por la revista de Obra del Grupo Editorial Expansión (2011).

\section{MÉTODOS Y TÉCNICAS DE INVESTIGACIÓN}

El mismo Plan Nacional de Desarrollo 2007-2012, señala que se han llevado a cabo medidas para eliminar distorsiones en materia de competencia, para propiciar una mejora regulatoria que reduzca los costos de apertura y operación de los negocios, y para favorecer el desarrollo de un sistema financiero eficiente que facilite el acceso al financiamiento en condiciones más competitivas. De esta manera, se coadyuva a impulsar un crecimiento más dinámico de las actividades productivas y un mayor nivel de bienestar de la población.

Para lograr ese nivel de bienestar en las 


\section{Cuadro 4. Catalogo de Actividades en orden de impacto en la economía en VENTAS}

\begin{tabular}{|c|c|}
\hline No. & Actividad \\
\hline 1 & Holding \\
\hline 2 & Vivienda económica y de interés social \\
\hline 3 & Vivienda media y residencial \\
\hline 4 & Edificación comercial, oficinas y servicios \\
\hline 5 & Edificación de naves y plantas industriales \\
\hline 6 & Obras para el tratamiento y distribución de agua, drenaje y riego \\
\hline 7 & Construcción de sistemas de distribución de petróleo y gas \\
\hline 8 & Construcción de Plantas de Refinería y Petroquímica \\
\hline 9 & Construcción de Obras para telecomunicaciones \\
\hline 10 & Obras de generación y conducción de energía eléctrica \\
\hline 11 & División de terrenos y construcción de obras de urbanización \\
\hline 12 & Carreteras, caminos, puentes y similares \\
\hline 13 & Obras para transporte eléctrico y ferroviario \\
\hline 14 & Construcción de presas y represas \\
\hline 15 & Obras marítimas, fluviales y subacuáticas \\
\hline 16 & Otras obras de ingeniería pesada o civil \\
\hline 17 & Construcción especializada en montaje de estructuras y trabajos en exteriores \\
\hline 18 & Cimentaciones, montaje de estructuras prefabricadas y trabajos en exteriores \\
\hline 19 & $\begin{array}{l}\text { Instalaciones y equipamiento en construcciones (eléctricas, hidrosanitarias, gas aire } \\
\text { acondicionado y calefacción) }\end{array}$ \\
\hline 20 & $\begin{array}{l}\text { Trabajos en acabados en edificaciones (colocación de muros falsos, aislamiento y } \\
\text { enyesado, cubrimientos de paredes, colocación de pisos) }\end{array}$ \\
\hline 21 & Otros trabajos especializados para la construcción \\
\hline
\end{tabular}

Fuente. Elaboración propia con base en los datos generados por la revista de Obra del Grupo Editorial Expansión (2011).

PYMES es que se deben cumplir las principales características del Sistema Tributario, que de acuerdo a Carrasco (2003, p. 65) son:

a) Máximo Sacrificio. Significa que debe recaudarse de los contribuyentes con la finalidad de atender los servicios públicos máximos necesarios.

b) Equilibrio. Es una hacienda del gasto, en la que se debe procurar y guardar un equilibrio entre el presupuesto y su ejercicio.

c) Perdurable. En virtud de que el erario público existirá mientras subsista el Estado. d) Coactiva. Puesto que los contribuyentes de un Estado no pueden eximirse del pago de las contribuciones o aprovechamientos.

e) Dependiente. Porque el titular de la hacienda pública debe acatar lo ordenado por el Poder Legislativo en la Ley correspondiente.

Pero analizando las características desde la perspectiva de Faya (1999), el sistema fiscal debe tener esto, porque este autor al igual 


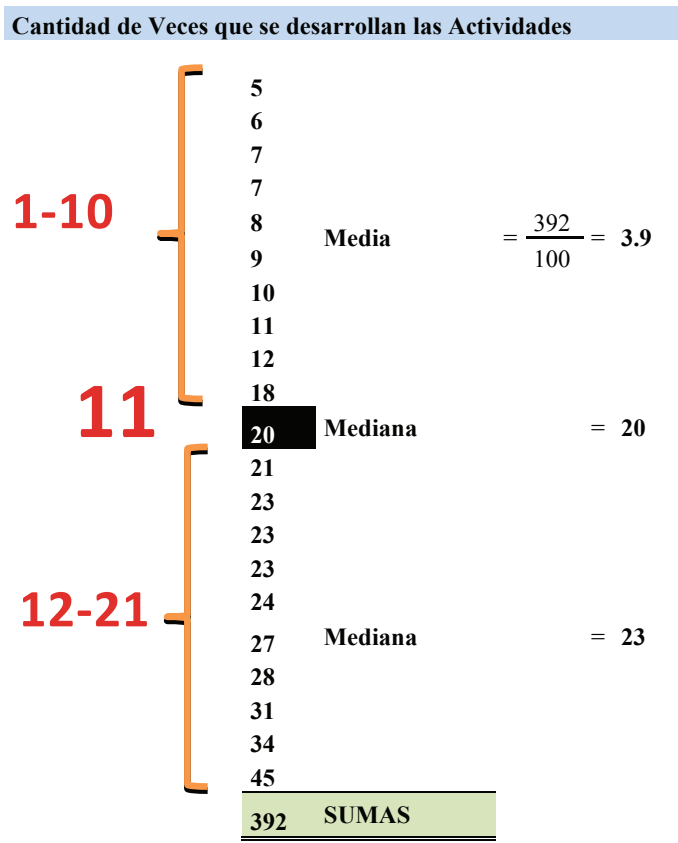

Figura 1. Determinación de la Media, Moda y Mediana.

que otros más se oponen a las reformas estructurales del sistema impositivo, puesto que el sistema fiscal debe ser: flexible, eficiente, abierto y coordinado .

Como punto de referencia para definir el método y técnicas de investigación revisamos de manera estadística que la media de las actividades que las 100 Constructoras más importantes desarrollaron nos genera un número cuatro, y se obtiene de dividir las 392 actividades llevadas a cabo entre 100 empresas Constructoras, su mediana es veinte (representado por el número que corresponde al elemento 11, pues se encuentra en el centro de las veces que las 21 actividades se llevan a cabo; y por último la moda es veintitrés, pues es el número de veces que tres actividades se repite en el muestreo de las 100 empresas. Esto se muestra en la figura 1 .

Después de analizar lo anterior, el método utilizadoparaestainvestigación fue elmétodo cuantitativo, por mostrar que esta naturaleza tiene como finalidad asegurar la precisión y el rigor que requiere la ciencia, según lo señalado por Rodríguez (2010, p.32). Esta creencia se vincula con lo señalado por Usher (1996, p. 12) quien explica que el mundo es objetivo e independiente de las personas que lo conocen, pues está constituido por fenómenos que siguen una ley y un orden, y que debemos dejar muy clara la separación entre los sujetos y objetos, así como entre hechos y valores, donde el mundo social es similar al mundo natural, por lo tanto existe orden y razón en él, y donde las ciencias naturales y sociales comparten una lógica común.

Cantidad de Veces que se desarrollan las Actividades

La población o universo muestral se compone de empresas de la Construcción organizadas y registradas en la Cámara Mexicana de la Industria de la Construcción de Culiacán , y las unidades de análisis son los profesionistas (contadores, auditores, fiscalistas y administradores) que en las empresas tienen relación directa con el

Fuente. Propia elaborada con base en los datos generados por la revista de Obra del Grupo Editorial Expansión (2011).

cumplimiento de las obligaciones en materia de IMSS y en forma especifica con el Reglamento del Seguro Social obligatorio para los trabajadores de la construcción por

$\begin{array}{lc}\begin{array}{l}\text { Cuadro 5. Capacitación } \\ \text { entrevistados en 2011. }\end{array} & \text { recibida de los sujetos } \\ \text { Criterio } & \% \\ \text { NULA } & 30.43 \% \\ \text { Deficiente } & 17.39 \% \\ \text { Regular } & 13.04 \% \\ \text { Buena } & 30.43 \% \\ \text { Excelente } & 8.70 \% \\ \text { Fuente: propia elaborada en base a los resultados } \\ \text { de la aplicación de los cuestionarios. }\end{array}$




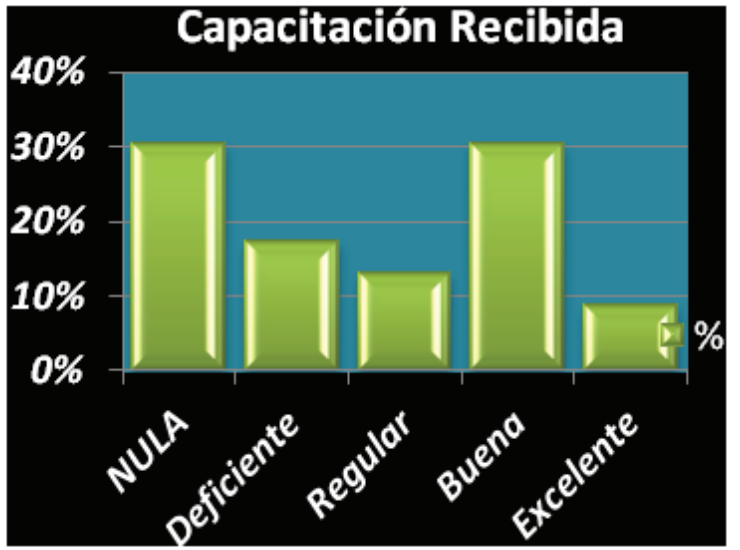

Figura 2. Capacitación recibida de los sujetos entrevistados en 2011.

Fuente. Propia elaborada en base a los resultados de la aplicación de los cuestionarios.

obra o tiempo determinado.

La muestra es de tipo probabilístico donde el tamaño de la población $(\mathrm{N})$ es de 600 empresas de la Construcción registradas en la CMIC, la estimación de valor de $\bar{Y}$, representada con la letra $\bar{y}$, es de 1 , y el valor de la desviación estándar o error máximo aceptable (se) es de 0.05 , y el valor del porcentaje estimado de la muestra o probabilidad de que ocurra el fenómeno es de 0.95 , con cuyos datos determinamos primero el valor de la varianza de la muestra (s2), dando como resultados 0.0475 (resultado de utilizar la fórmula $\mathrm{p}(1-\mathrm{p})$ ). Posteriormente determinamos el valor de la varianza de la población al cuadrado (que se obtiene de elevar al cuadrado el valor de "se", dando como resultado 0.0025). Esta información sirve de base para determinar el valor de la muestra sin ajustar donde $\mathrm{n} 1$ = s2 / V2; cuyo se sustituye en la fórmula de tamaño de la muestra o subconjunto de población $n=n 1 /((1+(n 1 / N))$; y al hacer

Cámara Mexicana de la Industria de la Construcción, máximo órgano de representación del gremio, la cual se encarga de consolidar, unificar y promover desde 1953 a las empresas afiliadas del país, otorgándoles servicios de calidad de representación y gestión, información, asesoría, capacitación, servicios educativos, certificación, y desarrollo tecnológico la sustitución nos da como resultado que debemos hacer 18 cuestionarios, y así se hizo, pero en forma adicional se aplicaron 5 entrevistas a diferentes profesionistas quienes tienen dentro de sus funciones la administración del ciclo de SATIC.

Entre las técnicas o instrumentos de medición que tenemos en el método cuantitativo utilizamos el cuestionario con un total de 15 preguntas de las cuales 5 de ellas son preguntas abiertas, dos de las cuales son clasificadas como demográficas o de ubicación del sujeto, con la finalidad de obtener información útil para la investigación, cuidando los aspectos del sentir en el participante que pudiera desvirtuar las respuestas proporcionadas, por lo que se respetó el hecho de no señalar nombre de los participantes en el mismo y así lograr mayor confianza y objetividad; el resto de preguntas fueron cerradas encaminadas a dar respuesta a la variable principal de los costos no identificados por fase en construcción de obra ejecutada no subcontratada con terceros.. La escala de medición utilizada en las preguntas del cuestionario quedaron en uso de dos preguntas con una escala de medición de actitud, dos de ellas se midieron con una escala de Likert y la última con una escala de diferencial semántico.

El método de administración de los

\begin{tabular}{llc|}
\hline $\begin{array}{l}\text { Cuadro 6. Percepción de los sujetos cuando son revisados } \\
\text { por personal del IMSS y su apreciación respecto a su } \\
\text { Reglamento. }\end{array}$ & \\
Percepción sobre: & Categoría & $\%$ \\
\hline Revisiones/Auditorías & Muy Profesionaly de acuerdo al RIMSS & $23.08 \%$ \\
& Justo & $15.38 \%$ \\
& De acuerdo al RIMSS & $15.38 \%$ \\
& Injusto & $15.38 \%$ \\
Normatividad & Se extralimitan & $30.77 \%$ \\
& Nula & $4.35 \%$ \\
& Deficiente & $26.09 \%$ \\
& Regular & $43.48 \%$ \\
Fuente propia: & Buena & $17.39 \%$ \\
cuestionarios. & Excelente & $\mathbf{8 . 7 0 \%}$ \\
& & \\
\hline
\end{tabular}


cuestionarios se constituyó con dieciocho de ellos aplicados de manera Autoadministrada de manera individual, tres entrevistas personales, uno administrado vía telefónica y el último por correo electrónico. El 61\% de los encuestados se desempeñan como Contadores de las empresas, el 17\% son Administradores, el 13\% Auditores y el 9\% Fiscalistas. Uno de los criterios de inicio para seleccionar a los sujetos que participaron de la muestra en nuestra población es que su desempeño laboral tuviera relación directa

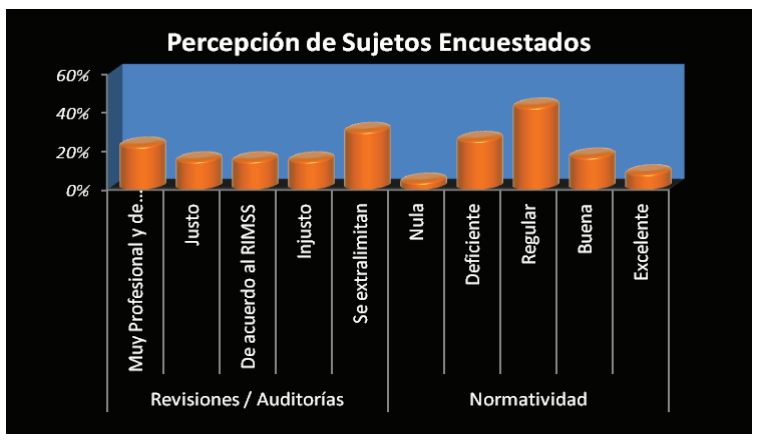

Figura 3. Percepción de los sujetos cuando son revisados por personal del IMSS y su apreciación respecto a su Reglamento.

Fuente. Propia elaborada en base a los resultados de la aplicación de los cuestionarios.

con el proceso de aplicación del RIMSS, pues esto permite lograr un resultado más coherente con los objetivos que nuestra investigación tiene.

A su vez, y a través del análisis de información recibida permitió elaborar una guía de entrevista, la cual se aplicó de manera personal (cara a cara) a los sujetos que participaron, entre los que se encuentran Contadores que laboran en despachos y en empresas de la construcción en Culiacán, quienes están en contacto directo con la responsabilidad y obligación de llevar a cabo las diversas etapas que comprenden y cierran con la etapa comprobación de los costos de obra por etapa cuando dichas obras no son contratadas con terceros.

\section{RESULTADOS Y DISCUSIÓN}

En promedio la antigüedad de los sujetos que participaron en la aplicación de los cuestionarios tiene tres años desarrollando sus actividades en las organizaciones que fueron parte de la muestra y considerando el número de personas que laboran en el área administrando el ciclo de cumplimiento en la materia son de uno punto tres. El 74\% de las empresas encuestadas se dedican a la construcción de vivienda económica o de interés social, mientras que el $13 \%$ eran empresas que tienen como giro la construcción de vivienda media o residencial, por otro lado el $9 \%$ se especializan a la construcción de naves o plantas industriales, y el $4 \%$ tenían un giro diferente. Esto de cierta manera apoya el criterio de que según el análisis de las 100 empresas constructoras más importantes alrededor del $40 \%$ de los ingresos de las mismas se generan en las empresas con giro de construcción de vivienda económica o de interés social.

De los sujetos entrevistados el 13\% no le tocó el proceso de trabajo de acuerdo a la normatividad del IMSS., cuando el documento que se llenaba era la AFIL, el 9\% le tocó conocer solamente el proceso de AFIL pero no trabajo en este, pero si lo ha realizado con el de SATIC y el resto que se compone del 78\% ha trabajado con ambos procesos. La experiencia que los profesionistas que participaron en la investigación han tenido en ambos procesos genera confianza en los resultados obtenidos.

El cuadro número 5 y la figura número 2 muestran aspectos relacionados con la capacitación, la cual fue recibida de revistas especializadas con un 13\%, de capacitación interna un 19\% (cursos recibidos de otras 
personas de la organización), asistiendo directamente a cursos un $25 \%$, capacitación recibida directamente del personal del IMSS un $6 \%$ y un $37 \%$ de consultas directas con asesores pagados a través de esquemas de outsourcing en el área de consultoría de IMSS.

Al revisar en las empresas encuestadas cuantas de ellas han estado en alguna revisión por parte del Instituto Mexicano del Seguro Social, para comprobar que los pagos efectuados cumplen con lo estipulado en el Reglamento del IMSS para los trabajadores de la construcción por obra o tiempo determinado y la respuesta obtenida en la investigación efectuada es que el $56 \%$ de las que han sido sujetas a alguna revisión, sus profesionistas han formado una percepción basados en la forma en que dichas revisiones han sido efectuadas, resumiendo todo como a continuación se detalla: tres empresas consideran que el trato es muy profesional y de acuerdo a las facultades que las mismas leyes y reglamentos les proporcionan, seis empresas coinciden en tres criterios como son muy profesional, de acuerdo a las facultades que tienen, e injusto (dos de las trece para cada criterio), y cuatro de ellas consideran que se extralimitan al llevar a cabo procedimientos como el exigir el pago de los adeudos de las empresas con quienes tienen contratos de obra para realizar trabajos, aún cuando dichas empresas cumplieron al $100 \%$ con los requisitos que el mismo reglamento contempla, así como algunas de ellas también fueron sujetos de cierre temporal de la obra en la presión de que cumplan con sus requerimientos.

De acuerdo a las respuestas y el cuadro anterior vemos que el $26 \%$ de las personas que participaron consideran que en efecto la normatividad respecto a la reglamentación para alta de obra y lo que el reglamento señala para comprobar a las autoridades el cumplimiento de pago correcto y completo, el $43 \%$ lo considera regular y sólo el $7 \%$ considera que si cumple con la forma en que los contribuyentes requieren para hacer frente a sus obligaciones.

Actualmente doce de las vientres empresas que forman parte de la muestra consideran que por manejar dos o más etapas, el costo de la nómina actualmente lo están identificando al separar los trabajadores por etapa aun cuando ellos pueden trabajar en etapas diferentes, siete de ellas prorratean el costo de nómina con la finalidad de poder comprobar a las autoridades, pero el Reglamento no tiene esta posibilidad actual.

\section{CONCLUSIONES}

Se hace necesario valorar la forma en que actualmente el Reglamento detalla la manera de cumplir con las obligaciones que el mismo señala a los contribuyentes cuando se tiene trabajadores eventuales por obra o tiempo determinado, puesto que actualmente no queda claro para los contribuyentes como se debe comprobar cuando la obra no se subcontrata con terceros, ya que la apreciación que los mismos contribuyentes manifestaron en las entrevistas es que podrían hacer modificaciones al reglamento actual con la finalidad de permitir se haga contabilización por periodos que pudieran ser anuales donde se puedan acumular las altas de obras que se hayan efectuado en dichos periodos y poder lograr un amarre, tal y como se hace con otro tipo de declaraciones de impuestos federales o los mismos impuestos de seguridad social y con esto permitiría lograr mayor claridad, ya que como se ha comentado los mismos cambios en los mercados en el sector de la construcción vuelve difícil y complicado llevar el control solo con altas por fases de construcción. 
Otra de las opciones consideradas es que el reglamento señale la posibilidad de llevar la nómina por cada etapa y por último como tercera opción es que permita el mismo prorrateo que actualmente se lleva. El 44\% de los contribuyentes lleva controles en Excel, el $31 \%$ se remonta a la contabilidad cuando hay necesidad de hacer aclaraciones o mostrar información al Instituto Mexicano del Seguro Social al momento de comprobar los costos de las obras, un 18\% lleva controles manuales adicionales, pero ve muy complicado cuando por cuestiones inherentes a la misma tecnología los archivos se dañan, y solo un 7\% tienen algún sistema hechizo adicional que les permita llevar los controles necesarios.

Sin lugar a dudas hacer modificaciones al reglamento no es una tarea fácil, pero conociendo la problemática que el Instituto tiene en estos momentos, se vuelve necesario tomar medidas que permitan lograr claridad y con eso los contribuyentes podrán estar en condiciones de cumplir con el pago de las contribuciones en tiempo y forma, que a su vez, ayudará a limpiar sus finanzas del IMSS, y en ese tenor consideramos que la tercer opción que consiste en y con esto se logró determinar que en efecto la percepción de estas PYMES es que se requiere dejar un criterio estándar para su comprobación a través del prorrateo de costos de manera proporcional en base a las fechas de contratación y fechas de término de las obras llevando un control que identifique costos de manera global, dejando un número de identificación en los papeles de trabajo para futuras revisiones por parte del Instituto Mexicano del Seguro Social.

\section{LITERATURA CITADA}

Carrasco Iriarte Hugo. (2003). Glosario de términos fiscales, aduaneros $\mathrm{y}$ presupuestales. México. Editores Iure. Segunda Edición Cámara Mexicana de la Industria de la Construcción http://www.cmic. org/intercmic/acerca.htm. Faya, V. Jacinto. (1999). Finanzas Públicas México. Editorial Porrúa, México.

González Fajardo José Antonio. (2010). Estudio de Administración efectiva de proyectos de construcción en el contexto de las Pymes. México. Página de internet. Fecha de consulta (2010, 14 de octubre). http://www. google.com.mx/search?hl=es \&so urce $=$ hp \&q $=$ pymes + de + la + constru ccion $\&$ meta $=\& a q=f \& a q i=\& a q l=\&$ oq $=\& g s \_r f a i=\& r l z=1 \mathrm{~W} 1 \mathrm{RNTN}$ _es http://www.cnnexpansion.com/ obras/2009/02/05/las-empresasmas-importantes. Las 100 Constructoras más importantes. Publicado el día 08 de junio del 2010. Revista Obra del Grupo Editorial Expansión. Bajado de internet el día 21 de diciembre del 2011. http://www.e-local.gob.mx/ work/ templates/enciclo/sinaloa/ mpios/25006a.htm Información de Colindancias del Municipio de Culiacán. Revisada el día 25 de Noviembre del 2011. Ley del Seguro Social (2010).

Lozano Carrillo Oscar, Cisneros Martínez Luis Felipe y Niebla Zataraín Juan Cayetano. (2009). Empresa familiar (casos). México. Grupo Editorial HESS, SA de CV. México. Documento de trabajo No. 65 del Centro de Estudios Sociales y de Opinión Pública de marzo del 2009 México. Ley del Seguro Social. México. Reglamento de la Ley del Seguro Social para los Trabajadores Eventuales por Obra o Tiempo 
Determinado. México. Plan Nacional de Desarrollo 2007-2012. OCDE. Organización para la Cooperaciónyel Desarrollo Económico. http://www. oecd.org/pages/0,3417,es_36288966 _36288120_1_1_1_1_1,00.html.

Rodríguez Valencia, Joaquín (2001). Como administrar pequeñas $\mathrm{y}$ medianas empresas. México. ECAFSA Thompson Learning.

Rodríguez Peñuelas Marco. (2010). Métodos de investigación. Diseño de proyectos de desarrollo de tesis en ciencias administrativas, organizacionales y sociales. México. FCA-UAS.

Rojas Juan A. (2003). On the interaction between Education and Social Security. Trabajo del Departamento de Economía. Universidad Carlos III de Madrid. 6E http://docubib. uc3m.es/WORKINGPAPERS/WE/ we033511.pdf

Rojas Juan A. (2002). Privatizing social security; the role of imperfect substitution between less and more experienced workers. Trabajo del Departamento de Economía. Universidad Carlos III de Madrid. 4E http://docubib. uc3m.es/WORKINGPAPERS/WE/ we022004.pdf http://www.e-local. gob.mx/work/templates/enciclo/ sinaloa/mpios/25006a.htm

Usher, R. (1996). A critique of the neglected epistemological assunptions of educational research. En D. Scott y R. Usher (Eds.), Undestanding educational research. Londres: Routledge.

\section{AGRADECIMIENTOS}

En nuestra humilde opinión, agradecemos a los lectores de este artículo; y ponemos a disposición las modestas aportaciones de quienes suscriben estas líneas del conocimiento. En el anhelo que a partir del mismo sea referente para seguir explorando en el campo de los estudios fiscales y financieros, de toda actividad propicia de desarrollo para la región y el país. Atendiendo el correcto análisis de la norma que debe propiciar el crecimiento ordenado y regulado de los diferentes sectores del país.

Dr. Rubén Antonio González Franco Profesor e investigador de tiempo completo titular "B" y Coordinador General de Investigación y Posgrado de la Facultad de Contaduría y Administración de la Universidad Autónoma de Sinaloa, Campus Culiacán, miembro del cuerpo académico en Formación "Estudios Fiscales y Administrativos" UAS-CA-256, evaluador de ANFECA, Doctor en Estudios Fiscales del PNPC FCA-UAS CONACYT (mención honorífica), Maestría en Auditoría por la UNAM (mención honorífica), Contador Público certificado por la FNAMCP y el IMCP. Dirección: Santa María No. 2290, Fracc. Providencia, C.P. 80290, Culiacán Sinaloa. Teléfono: 0166716306 14, Email: ruben0304@hotmail.com

Jesús Manuel Meza Valdéz

Profesor de cátedra de Instituto Tecnológico de Estudios Superiores Campus Culiacán, de Tec Milenio Campus Culiacán, de Universidad de Durango y de la Facultad de Contaduría y Administración [FCA] (modalidad presencial y a distancia.-SUAD), Coordinador de la Licenciatura en Gerencia de Tiendas de la FCA, así como Estudiante del Doctorado en Estudios Fiscales de la FCA de la Universidad Autónoma de Sinaloa, en programa de calidad de CONACYT. San Agustín No. 58 Desarrollo Industrial La Primavera, Culiacán, Sinaloa, México, 
C.P. 80300 Tel. 00526677440286 correo electrónico:jmanmeza_@hotmail.com.

Dr. Rubén Miranda López

Profesor e investigador de tiempo completo titular "C" Director de la Facultad de Contaduría y Administración de la Universidad Autónoma de Sinaloa, Campus Culiacán, miembro del cuerpo académico en Formación "Estudios Fiscales y Administrativos" UAS-CA-256, evaluador de ANFECA, Doctorante en Estudios Fiscales del PNPC FCA-UAS CONACYT, Maestría en Auditoría por la UNAM (mención honorífica), Contador Público certificado por la FNAMCP y el IMCP. Dirección: Ciudad Universitaria, Culiacán Sinaloa. Teléfono: 01667 2240599, correo electrónico: fcarmirandagmail.com. 